RECENSIÓN

\title{
La economía invisible y las desigualdades de género
}

\author{
The invisible economy and the gender inequalities
}

\begin{abstract}
Ana María Gálvez González*
Doctora en Ciencias de la Salud. Escuela Nacional de Salud Pública. La Habana, Cuba.
\end{abstract}

\section{La importancia de medir y valorar el trabajo no remunerado}

Bajo este título se presenta un interesante libro que integra un conjunto de trabajos sobre la medición y valoración del tiempo dedicado por los miembros del hogar a actividades productivas que se mantienen invisibles desde el punto de vista de la contabilidad nacional regida por el Sistema de Cuentas Nacionales de 1993. Los trabajos fueron elaborados por un conjunto de autores, bajo los auspicios de la Organización Panamericana de la Salud, en coordinación con la Comisión Económica para América Latina y El Caribe y el Consejo Superior de Investigaciones Científicas de España, entre los años 2001 y 2006.

El libro consta de 285 páginas y está compuesto por tres partes. La primera presenta un análisis de las cuentas satélite del sector hogares y la medición y valoración del trabajo no remunerado, sus resultados e implicaciones de política para la reducción de las desigualdades de género. Este segmento del libro abarca sus seis primeros capítulos. En ellos se incluyen resultados empíricos que muestran el valor que tendría la producción realizada con trabajo no remunerado en relación con el Producto Interno Bruto, según diferentes enfoques, criterios de medición y valoración. Se presentan estimaciones para países y se incluyen propuestas metodológicas para el uso de las Cuentas Satélite del Sector Hogares. Resultan interesantes los resultados empíricos para la experiencia de España, donde además se hace un análisis de las implicaciones sobre las desigualdades de género derivadas de las estimaciones sobre la magnitud y distribución entre hombres y mujeres de la carga de los cuidados de salud y del cuidado de otros miembros del hogar.

La segunda parte del libro, capítulos del 7 al 11, está dedicada a la presentación de propuestas conceptuales y metodológicas. Sin embargo, es preciso llamar la atención acerca de que este tipo de enfoque, en alguna manera, había sido abordado anteriormente. En esta parte de la obra lo distintivo es que aparecen 
reflejados temas relacionados con las Encuestas del Uso del Tiempo. Se presenta una síntesis de los enfoques y resultados de la aplicación de estos instrumentos en Bolivia, Ecuador, Guatemala, México y Nicaragua. Los trabajos presentan la forma de utilizar estas encuestas en la evaluación de las desigualdades de género que resultan de la distribución de la carga del tiempo de trabajo no remunerado dedicado a actividades domésticas. Muy interesantes resultan dos trabajos que resumen experiencias derivadas de estas investigaciones así como los retos desde el punto de vista conceptual y metodológico de la aplicación de los conceptos de "cuidado de los demás" y de "cuidado de los niños" en países de habla inglesa.

Por último se presenta la tercera parte del libro donde se resumen experiencias y resultados a lo largo de los capítulos 12 y 13. Estos analizan los sesgos de género que yacen en la base de los conceptos económicos de la contabilidad nacional y las estadísticas económicas de los países. Se plantean conceptos y métodos para destacar la importancia para el desarrollo y bienestar del trabajo no remunerado que realizan las mujeres y hombres fuera del mercado. Especialmente interesante en este capítulo es la propuesta de ampliación del concepto de Población Económicamente Activa con vistas a incluir el trabajo no remunerado que queda fuera del mercado. Otro tema que merece atención, es la propuesta de un Índice de Progreso Genuino como medida alternativa al Producto Interno Bruto ampliado, obtenido a partir de las cuentas satélites de la producción del sector hogares.

El libro presenta 169 citas bibliográficas referenciadas en el texto, es decir, un promedio de algo más de 12 citas para cada uno de los 14 trabajos incluidos. Aunque esta proporción podría parecer un tanto reducida, es preciso señalar que las mismas provienen de instituciones y autores con reputación en el tema. Una buena parte está disponible en Internet, lo que facilita el acceso a aquellos que deseen profundizar o completar conocimientos.

Esta obra revela las relaciones económicas asociadas a las desigualdades de género y se erige como una invitación a la producción de trabajos científicos encaminados a analizar ese tipo de desigualdades que existen en diferentes países. Por otra parte, el libro es un punto de partida para el desarrollo de nuevas bases de información e indicadores que resalten el valor de la contribución del trabajo no remunerado de las mujeres al bienestar y desarrollo económico de los países.

El título del libro pudiera hacer pensar en una obra dirigida básicamente a estudios de temas de economía o a especialistas en desigualdades de género, sin embargo, la riqueza de sus textos, la seriedad en la forma de abordar los conceptos, la coherencia metodológica, el buen uso de las herramientas de la estadística económica y la proyección hacia la formulación de políticas encaminadas a la reducción de las desigualdades de género, incitan a recomendar la obra a un grupo más amplio de lectores, en particular todo aquel personal dedicado a la docencia, investigación y toma de decisiones en el contexto de las ciencias de la salud.

* Jefa del departamento de Economía de la Salud de la Escuela Nacional de Salud Pública. 